\title{
Numerical simulation of long rods impacted by particle beams
}

\author{
Marcus Portelli* \\ CERN, CH-1211 Geneva 23, Switzerland, and University of Malta, Msida MSD2080, Malta
}

\author{
A. Bertarelli, F. Carra, and L. K. Mettler \\ CERN, CH-1211 Geneva 23, Switzerland \\ P. Mollicone and N. Sammut \\ University of Malta, Msida MSD2080, Malta
}

(Received 7 November 2017; published 25 June 2018; corrected 19 October 2018)

\begin{abstract}
Analytical solutions detailing the propagation of longitudinal waves in slender rods subjected to a sudden increase of internal energy provide simple tools for the calculation of the temperature distribution in impacted rods as well as the resulting mechanical response. The topic is of great interest in particle accelerator technology, especially with regards to collimation systems, where beam intercepting devices can be generally approximated to one-dimensional (1D) elements potentially subjected, in accidental scenarios, to abrupt thermal energy depositions induced by the impacting particles. In this study, two finite element numerical models are presented and compared to the analytical solutions by Bertarelli, Dallocchio and Kurtyka, discussing the rapid temperature increase in slender rods due to particle beam impacts and the resulting dynamic longitudinal response. The first model is a sequentially coupled thermomechanical analysis; the second is based on a modal analysis to find the harmonic response of the system. The results indicate that phenomena neglected in analytical solutions, primarily dispersion of the longitudinal wave due to interactions with the free external surface of the rod, can be included in numerical models and can be observed in simulation results. The study further shows how numerical methods can be utilized to predict the frequencies and amplitudes of high-frequency disturbances in the longitudinal wave signal, and how these effects can be mitigated in preparation for experimental scenarios by fine-tuning the geometry of the rod and varying the duration of the pulse. This is especially useful with regards to experiments conducted in the HiRadMat facility at CERN, such as the recently conducted HRMT36 experiment, where highfrequency components can distort the signal to be observed.
\end{abstract}

DOI: 10.1103/PhysRevAccelBeams.21.063501

\section{ANALYTICAL MODELING OF THERMALLY INDUCED ELASTIC STRESS WAVES}

Subatomic particles interacting with matter transfer some of the energy to the material they interact with in the form of heat, leading to a temperature increase in the impacted material. Different effects may result from such an interaction, depending on the power density deposited. In particle accelerators, a continuous energy deposition is provoked by beam losses, which can last from a few seconds up to hours. In the case of accidental beam impacts, energy is rapidly deposited in the order of

\footnotetext{
*marcus.portelli.12@um.edu.mt

'Present address: University of California, Berkeley.
}

Published by the American Physical Society under the terms of the Creative Commons Attribution 4.0 International license. Further distribution of this work must maintain attribution to the author(s) and the published article's title, journal citation, and DOI. nanoseconds to microseconds, in turn leading to a dynamic response of the structure [1].

The dynamic response of slender rods subjected to a very fast internal temperature increase is of particular interest for high energy particle physics applications, as many beam intercepting devices are in the form of long rods of material. Studies on analytical solutions for this subject were performed by Bargmann [2] and Sievers [3], and more recently, work by Bertarelli et al. [4] has focused on both longitudinal and flexural vibrations. The latter is used as a general reference in this study, with emphasis on the longitudinal response.

Bertarelli [4] considers a slender cylindrical rod subject to a temperature increase induced by an internal heat generation along the rod axis. This is a common scenario when particle accelerator components are subject to high energy subatomic particle beams. Beam targets are one example, generally being slender rods directly hit by a beam in order to create a shower of secondary particles [5]. The rod is simply supported and free to expand at its 
extremities. The energy deposition has a Gaussian distribution over the cross section, with a standard deviation $\varphi$ and eccentricity $\eta$. The material model assumes an isotropic material with linear elastic behavior, no damping, and with thermal and mechanical properties independent of temperature.

As mentioned, in accidental beam impact scenarios the heating process typically lasts a few nanoseconds to microseconds, and during this thermal shock duration $\tau$, the deposited energy can be considered to grow linearly. If the thermal diffusion time $t_{d}$ is much longer than $\tau$, it can be assumed that no heat diffusion occurs during the thermal shock and that therefore the temperature increase is proportional to the deposited energy. The system can initially be considered adiabatic, hence the total energy remains constant following the energy deposition. Due to the rapidity of energy deposition, material inertia effects cannot be neglected, and in fact play a major role when considering longitudinal and flexural stresses. Material inertia in the radial direction, and consequently its effects on the longitudinal dynamic response, is neglected in the analytical model, based on considerations made by Graff [6]. As will be shown in this study, such effects have a significant impact on the longitudinal response and cannot be generally neglected.

The problem can be solved in two steps-a thermal analysis needs to be performed first, followed by a structural analysis. Assuming a constant temperature distribution along the rod axis with no eccentricity in the radial direction, the thermal analysis can be reduced to a onedimensional (1D) problem. For a specified Gaussian load distribution, the energy density $\left(\mathrm{J} / \mathrm{m}^{3}\right)$ across the crosssection at the end of the energy deposition, $U(r)$, takes the following form:

$$
U(r)=U_{\max } e^{-r^{2} / \phi^{2}},
$$

where $r$ is the radial position and $U_{\max }$ is the maximum energy density deposited, at the center of the beam. Assuming no heat diffusion occurs during the energy deposition and that the heat capacity is constant, the temperature increase across the cross section at the end of the thermal shock can be simply calculated by

$$
T(r)=\frac{U(r)}{\rho c_{p}} .
$$

The system is considered to be adiabatic, therefore the total energy following the beam impact remains constant and the final uniform temperature increase of the system is calculated by

$$
T_{f}=\frac{\int_{r} U(r) d r}{\rho c_{p} \pi R^{2}} .
$$

Due to the high energy and temperature gradient across the radius considered for the Gaussian distributed load, this can be simplified by instead considering a pencil beam, consisting of a circular cross section having a uniform load. In this case, for a beam without eccentricity, Eqs. (1)-(3) are simplified to

$U(r)=U_{\max } \quad$ for $r<\varphi \quad U(r)=0$ elsewhere,

$T(r)=T_{\max }=\frac{U_{\max }}{\rho c_{p}} \quad$ for $r<\varphi \quad T(r)=0$ elsewhere,

$$
T_{f}=T_{\max } \frac{\phi^{2}}{R^{2}},
$$

where $\varphi$ is the pencil beam radius and $R$ is the radius of the rod.

Thermal expansion is initially prevented by the body's inertia, establishing a coupling between thermally induced forces and inertia forces. In the case of a rod, this results in dynamic stresses propagating along the rod as elastic stress waves, with the system still being in a compressive state. The stress relaxation initiates from the two ends of the rod, as shown in Fig. 1, with two stress waves propagating along the rod and superimposing at the center, before reflecting at the opposite end.

For each propagating wave, a reference stress can be defined as follows:

$$
\sigma_{\mathrm{ref}}=E \alpha T_{f}
$$

This value corresponds to the compressive axial stress that would be induced by a uniform temperature increase $T_{f}$, in a rod with its ends fixed. This stress is equal to the maximum stress induced by each stress wave, resulting in a maximum dynamic longitudinal stress of $2 \sigma_{\text {ref }}$ when the two waves superimpose. This only occurs if the pulse duration is short enough, and with a longer energy deposition time this maximum stress value might not be reached. The evolution of the dynamic longitudinal stress scaled to the reference stress, as computed in Bertarelli's study [4], is shown in Fig. 2.

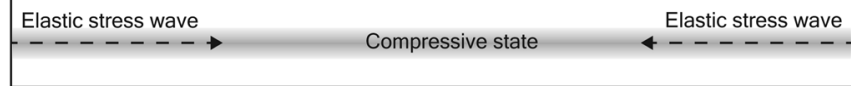

FIG. 1. Compressive state of the rod following the energy deposition, resulting in the propagation of longitudinal stress waves from its extremities. 


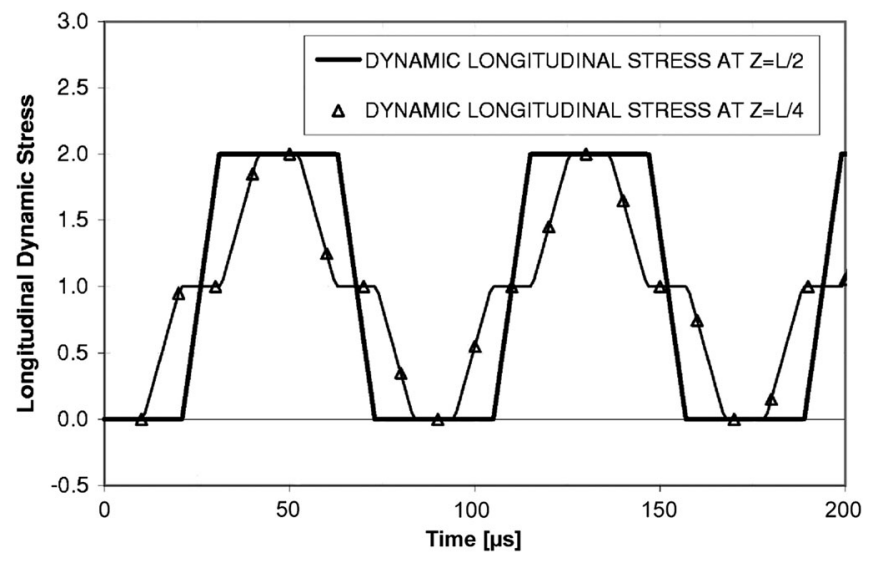

FIG. 2. Longitudinal dynamic stress at the center of the rod $(z=L / 2)$ and at one-fourth the length of the $\operatorname{rod}(z=L / 4)$ [4].

The first fundamental longitudinal period $t_{L}$ for elastic longitudinal waves is found by

$$
t_{L}=\frac{2 L}{c_{0}}
$$

where $c_{0}=\sqrt{\frac{E}{\rho}}$ is the velocity of longitudinal wave propagation in the material and $L$ is the length of the rod.

Another important parameter is the thermal diffusion time $t_{d}$, related to the time it takes to reach a uniform temperature distribution in a specified region by means of heat diffusion processes:

$$
t_{d}=\frac{B^{2}}{a},
$$

where $B$ is the relevant dimension (the radius in case of cylinders) and $a$ is the thermal diffusivity, given by

$$
a=\frac{k}{\rho c_{p}}
$$

where $k$ is the conductivity, $\rho$ is the density, and $c_{p}$ is the specific heat capacity of the material.

The time period for radial waves propagating from the center of the rod outwards in hydrostatic conditions is defined by

$$
t_{R}=\frac{2 R}{\sqrt{\frac{K}{\rho}}},
$$

where $R$ is the radius of the rod and $K$ is the bulk modulus of the material. The radial waves travel to the rod's free surface and are then reflected inwards.

In the analytical model considered, radial inertia effects on longitudinal waves are neglected based on the hypothesis that the frequency of the system is small compared to a reference value. This follows the study made by Graff [6] on the breakdown of the classical wave theory with regards to dispersive effects, showing that up to a limiting working frequency radial inertia can be neglected and the classical wave theory for longitudinal wave propagation in cylindrical rods is relevant. Beyond this frequency, the classical wave theory breaks down and radial inertia must be considered.

As described by Carra [7], there are two separate phenomena leading to high-frequency disturbances in axial oscillations, mainly cylindrical waves propagating from the center of the rod and dispersion of the longitudinal wave due to the boundary conditions.

The first phenomenon is related to radial waves propagating from the center of the rod (the point of impact), which travel to the external surface of the rod and are then reflected back. The radial displacement produced by these waves lead to an axial component related to the material's Poisson's ratio. For materials with a low Poisson's ratio, radial displacements are small and the Poisson effects on the axial vibrations are therefore not largely significant.

The second phenomenon is related to the dispersion of the longitudinal wave due to the free external surface of the rod. In this case, since the velocity of the wave is dependent on its frequency, higher frequencies generated by effects at the lateral free surface travel slower. Figure 3 shows the propagation of waves in a cylinder, with release waves generating at the free surfaces and trailing the main wave at lower velocities. This causes fluctuation in particle velocity, and is consequently observed in stress and strain readings at the surface of the rod.

Pochhammer [8] describes the phase velocity $c_{p}$ in the case of a longitudinal bar by

$$
\frac{c_{p}}{c_{0}}=1-\nu^{2} \pi^{2}\left(\frac{r}{\Lambda}\right)^{2}
$$

where $c_{0}$ is the ideal velocity, $\nu$ is the Poisson's ratio, $r$ is the bar radius and $\Lambda$ is the length of the wave, showing how dispersion is affected directly by the parameters listed, including the Poisson's ratio of the material.

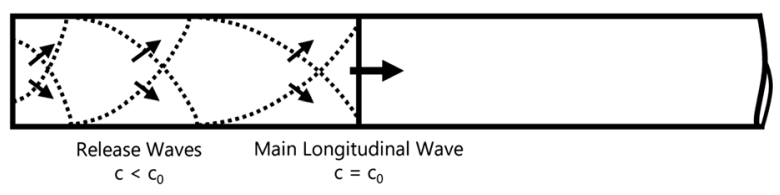

FIG. 3. Longitudinal wave propagation in cylindrical elastic bar, showing effects at free boundaries. 
TABLE I. Linear, temperature independent material properties for tungsten and graphite [10].

\begin{tabular}{lcccc}
\hline \hline Material property & Symbol & Tungsten & Graphite & Units \\
\hline Density & $\rho$ & 19300 & 1785 & $\mathrm{~kg} / \mathrm{m}$ \\
Specific heat capacity & $c_{p}$ & 130 & 1100 & $\mathrm{~J} / \mathrm{kgK}$ \\
Young's modulus & $E$ & 410 & 11.5 & $\mathrm{GPa}$ \\
Coefficient of thermal & $\alpha$ & $4.25 \times 10^{-6}$ & $4.5 \times 10^{-6}$ & $1 / \mathrm{K}$ \\
$\quad$ expansion & & & & \\
Poisson's ratio & $\nu$ & 0.28 & 0.1 & $/$ \\
Thermal conductivity & $k$ & 145 & 60 & $\mathrm{~W} / \mathrm{mK}$ \\
\hline \hline
\end{tabular}

\section{NUMERICAL MODELING OF THERMALLY INDUCED ELASTIC STRESS WAVES}

In this study, the problem presented is studied with a finite element model implemented in ANSYS. The problem is solved by first performing a transient thermal analysis to model the energy deposition during the beam impact, the results of which are input in a transient structural analysis as a thermal load to model the mechanical response of the system during and after the impact.

In the design phase of components subject to such loads, numerical simulations are preceded by a predesign based on analytical models such as that described in the previous chapter, which allows for the computation of temperatures and thermally induced stresses as defined by Eqs. (5)-(10). These preliminary calculations are then followed by numerical modeling, allowing to easily account for material nonlinearities with temperature, as well as other phenomena which cannot be modeled analytically, such as high-frequency effects on longitudinal waves and singularities at free mechanical boundaries.

The numerical model considers a rod with circular cross section, having a length of $125 \mathrm{~mm}$ and a diameter of $10 \mathrm{~mm}$. The particle beam is modeled by considering an internal heat deposition in a cylinder of radius $0.5 \mathrm{~mm}$ along the longitudinal axis of the rod, with a pulse duration of $7.2 \mu \mathrm{s}$ (a typical timescale in the case of beam impact on structures in CERN accelerators and experimental facilities, such as HiRadMat [9]) and a total deposited energy density of $1000 \mathrm{~J} / \mathrm{m}^{3}$ at the end of the pulse. The problem is modeled in $2 \mathrm{D}$, utilizing axial symmetry along the rod's longitudinal axis. For the thermal model, the initial temperature is taken to be $22^{\circ} \mathrm{C}$, with adiabatic boundary conditions. For the structural analysis, the rod is unrestricted and free to expand in any direction.

Two material models are considered in this section: tungsten and graphite, two materials which are widely used in accelerator technology. In both cases the materials are modeled with linear, temperature-independent properties, as is the case in the analytical model considered. The two materials have very different material properties, as indicated in Table I, with graphite having a relatively low Poisson's ratio, which theoretically minimizes Poisson's ratio effects resulting from the propagation of waves in the radial direction, and tungsten having a high density, stiffness, and Poisson's ratio.

The analytical model allows for the calculation of various values, such as the wave period, maximum temperature increase, and reference stress of the longitudinal wave, which can be compared with numerical results. Calculated results are shown in Table II for graphite and tungsten rods with the defined dimensions.

Temperature results can be compared with thermal analysis results computed in ANSYS. Figure 4 shows the numerically computed temperature distribution along the radius of the rods at different times. At the end of the energy deposition, the maximum temperature is $420.5^{\circ} \mathrm{C}$ and $531.3{ }^{\circ} \mathrm{C}$ for tungsten and graphite rods respectively, agreeing well with the analytical solutions for temperature increase of $398.6^{\circ} \mathrm{C}$ and $509.3{ }^{\circ} \mathrm{C}$, considering an initial temperature of $22^{\circ} \mathrm{C}$. As seen in Fig. 4, at the end of the energy deposition the maximum temperature is constant along the radius of the heated region, with a sharp drop to the initial temperature at the boundary. At the end of the energy deposition the heat starts diffusing throughout the body, until a final uniform temperature is achieved. Note that the temperature along the rod's longitudinal axis does not vary since the energy deposition is assumed constant along this axis.

The reference stress for the longitudinal wave is a function of the final uniform temperature in the rod and is calculated with Eq. (7). In the numerical model, the results from the thermal analysis are imported in the structural analysis as a thermal load. Figure 5 shows the dynamic longitudinal strain at the center of the tungsten and

TABLE II. Calculated values for speed of sound, longitudinal wave period, longitudinal wave frequency, maximum temperature increase, final temperature increase, reference stress, reference strain (reference stress divided by Young's modulus), and thermal diffusion time for tungsten and graphite rods with radius $5 \mathrm{~mm}$ and length $125 \mathrm{~mm}$.

\begin{tabular}{lcccc}
\hline \hline Calculated value & Symbol & Tungsten & Graphite & Units \\
\hline Speed of sound & $c$ & 4609 & 2538 & $\mathrm{~m} / \mathrm{s}$ \\
$\begin{array}{l}\text { Longitudinal wave period } \\
\text { Longitudinal wave }\end{array}$ & $t_{L}$ & 54.2 & 98.5 & $\mu \mathrm{s}$ \\
$\quad f_{L}$ & 18.4 & 10.2 & $\mathrm{kHz}$ \\
$\quad \begin{array}{l}\text { Maximumcy temperature } \\
\quad\end{array}$ & $T_{\max }$ & 398.6 & 509.3 & ${ }^{\circ} \mathrm{C}$ \\
$\quad$ increase & & & & \\
$\begin{array}{l}\text { Final temperature increase } \\
2 \times \text { reference stress }\end{array}$ & $T_{f}$ & 4.0 & 5.1 & ${ }^{\circ} \mathrm{C}$ \\
$2 \times$ reference strain & $2 \sigma_{\text {ref }}$ & 13.89 & 0.53 & $\mathrm{MPa}$ \\
Thermal diffusion time & $t_{d}$ & 33.9 & 45.8 & $\mu \mathrm{m} / \mathrm{m}$ \\
\hline \hline
\end{tabular}



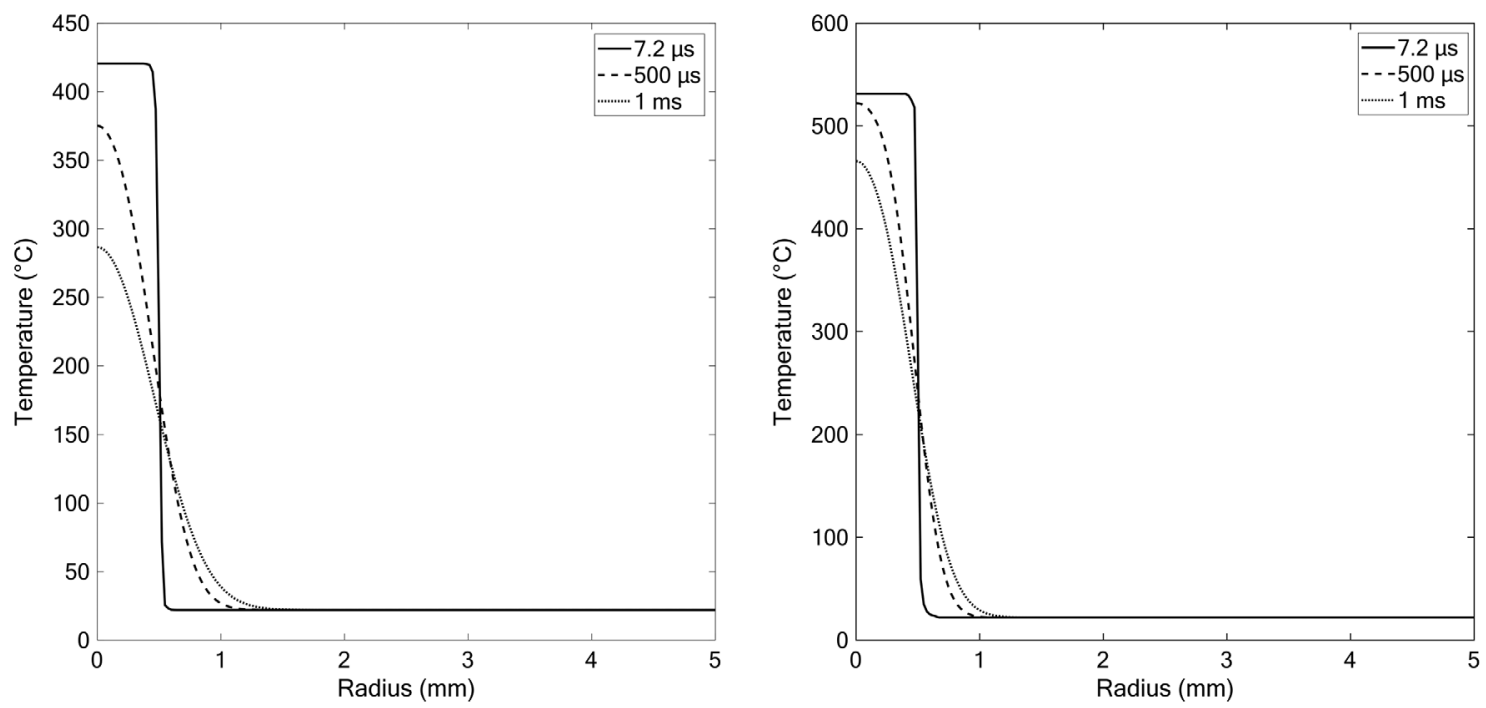

FIG. 4. Temperature distribution along tungsten rod (left) and graphite rod (right) radius at different times: $7.2 \mu$ s (at the end of the energy deposition), at $500 \mu \mathrm{s}$, and at $1 \mathrm{~ms}$.

graphite rods (at radial and longitudinal positions of $0 \mathrm{~mm}$ and $\mathrm{L} / 2$ respectively) computed numerically. The trapezoidal shape of the analytical solution for the longitudinal wave in the center of the rod, as shown in Fig. 2, is replicated well in the numerical results for both material models, as is the longitudinal wave period, however, the maximum dynamic strain is underestimated in the analytical calculations - this is mainly due to high-frequency disturbances overlapping the longitudinal wave signal. These disturbances are observable in results for both material models, and can be seen to increase in amplitude as the longitudinal wave propagates through the material.
A Fourier analysis of the results, shown in Fig. 6, identifies the frequency spectrum of the obtained signals, with frequencies in the range of $0-130 \mathrm{kHz}$ dominated by the odd harmonics making up the underlying longitudinal wave. At higher frequencies, peaks can be observed at 158 and $435 \mathrm{kHz}$ for the graphite material model. For the tungsten rod, two adjacent frequency peaks can be identified from the Fourier analysis at approximately 345 and $350 \mathrm{kHz}$, resulting in a beating effect in the signal.

There are various phenomena which can contribute to the generation of the high-frequency disturbances observed in the results shown in Figs. 5 and 6. The analytical model
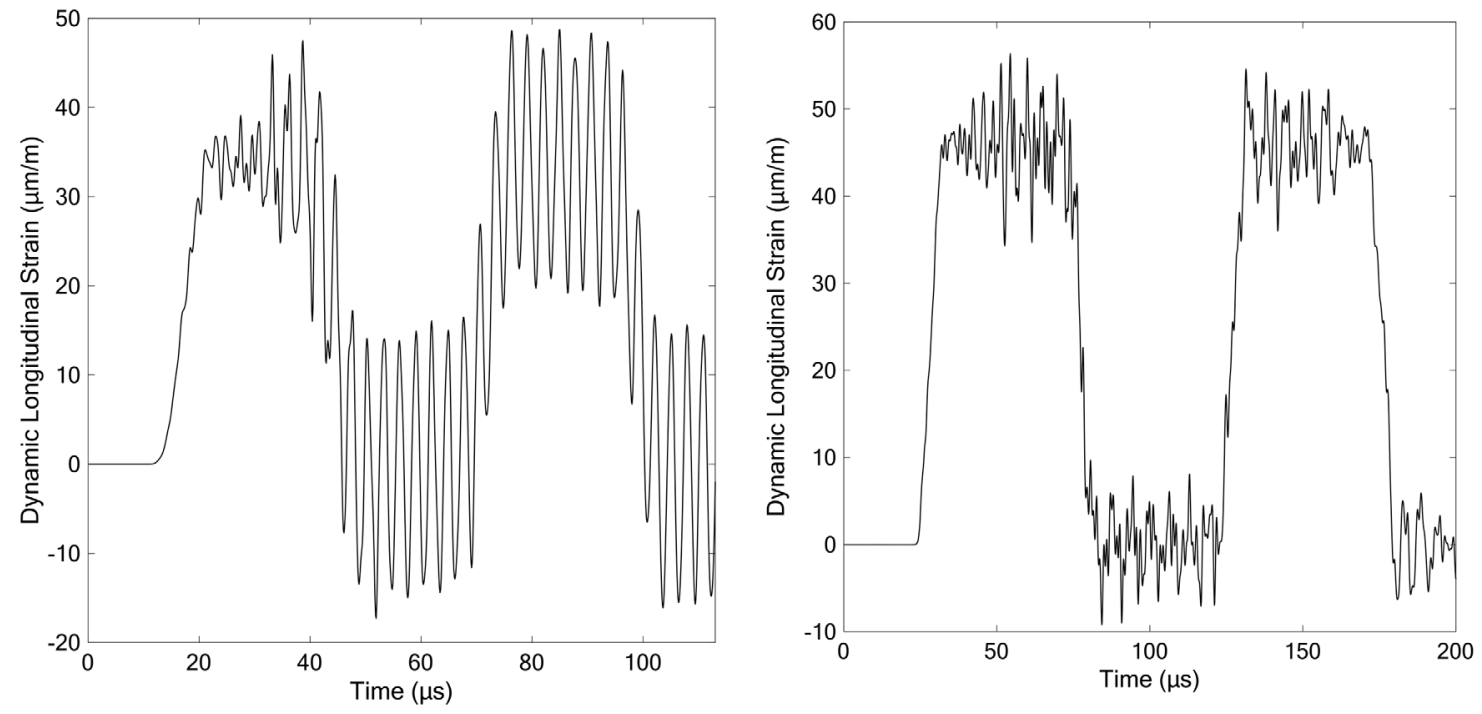

FIG. 5. Dynamic longitudinal strain for tungsten (left) and graphite (right) rods (at a radius of 0 mm and a longitudinal position of $\mathrm{L} / 2$ ). The computed maximum dynamic longitudinal strain $\left(2 \varepsilon_{\text {ref }}\right)$ is $33.9 \mu \mathrm{m} / \mathrm{m}$ for tungsten and $45.8 \mu \mathrm{m} / \mathrm{m}$ for graphite, while the simulation results show that the strain, while maintaining the expected trapezoidal shape, oscillates around this value for an actual maximum strain of approximately 49 and $56 \mu \mathrm{m} / \mathrm{m}$ for tungsten and graphite, respectively. 

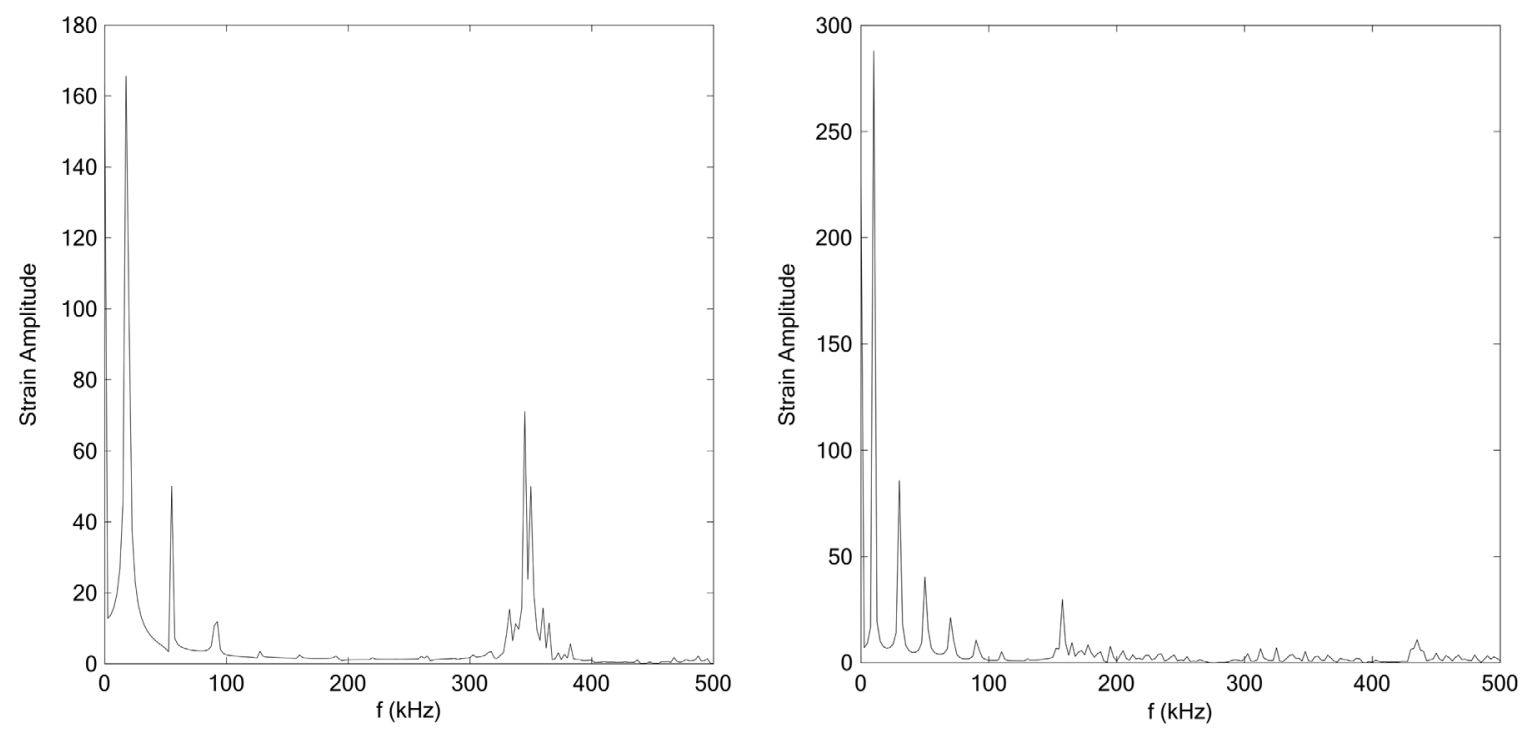

FIG. 6. Fourier analysis of the dynamic longitudinal strain results shown in Fig. 5 for tungsten (left) and graphite (right) rods with length $125 \mathrm{~mm}$ and radius $5 \mathrm{~mm}$. The frequency domain results show high-frequency peaks at $345 \mathrm{kHz}$ for tungsten and two peaks at 158 and $435 \mathrm{kHz}$ for graphite, whilst the lower frequencies are dominated by the odd harmonics making up the square longitudinal wave.

considered requires various simplifications, including neglecting inertia in the radial direction. The disregard of radial inertia in turn neglects lateral effects on the longitudinal waveform and effects due to wave dispersion. As seen in the results, neglecting such phenomena could lead to the underestimation of dynamic stresses induced by particle beam impacts, as the high-frequency radial components are observed to have a significant amplitude in certain cases, such as in the modeled tungsten rod, where the highfrequency components have an amplitude of more than $40 \%$ of the base longitudinal harmonic. This can result in a non-negligible increase in the maximum equivalent stress, leading to a nonconservative prediction of the dynamic stress, which can indeed have an impact in the design of beam-intercepting devices, where the calculated values must be compared with the allowable stress. This may potentially lead to the failure of the component due to the stress deviations caused by the high-frequency components.

The increase in stress can be even greater than what is observed in this case study, as shown in the HRMT27 (Rodtarg) experiment at CERN [11], where high-frequency radial waves with relatively large amplitudes were observed in the longitudinal displacement readings of impacted highdensity targets. In this case, the radial component was in fact the dominating phenomenon in the signal. Similarly, other experiments which plan to reproduce the behavior of components impacted by particle beams, such as the recently completed HRMT36 (MultiMat) [12], require the use of instrumentation having a high sampling rate in order to register the high-frequency signals. Better understanding of such phenomena ultimately benefits the design of full-scale collimators, which are required to operate safely upon impact of high intensity particle beams [13].

The above results prompt further studies on the parameters affecting the frequency and amplitude of highfrequency components in longitudinal waves. The work presented in this section aims to identify and better understand the phenomenon being observed, whilst the following section explores the dependence on parameters such as radius and length.

Two additional scenarios are now considered to better understand the factors affecting the high-frequency components observed in the dynamic longitudinal response of the rod, namely scaling the beam impact area over the whole rod section, and modifying the material properties and setting the Poisson's ratio to zero. This allows the separation of the different phenomena, i.e. lateral inertia effects on the longitudinal wave due to the Poisson's ratio and effects due to wave dispersion. All other numerical model parameters are retained, namely a rod length of $125 \mathrm{~mm}$ and a radius of $5 \mathrm{~mm}$, a Poisson's ratio of 0.28 for tungsten and 0.1 for graphite for the first scenario, and a beam impact diameter of $1 \mathrm{~mm}$ for the latter scenario.

By assuming a beam covering the whole cross section of the rod, maintaining the same total energy deposited, the rod is heated throughout its volume and therefore the final uniform temperature increase $\left(4.0^{\circ} \mathrm{C}\right.$ for the tungsten rod and $5.1{ }^{\circ} \mathrm{C}$ for graphite) is achieved at the end of the pulse, rather than after the thermal diffusion time elapses. In this case, as shown in Fig. 7 in the time domain and Fig. 8 in the frequency domain, the results still show a clear highfrequency component in the longitudinal wave signal for 

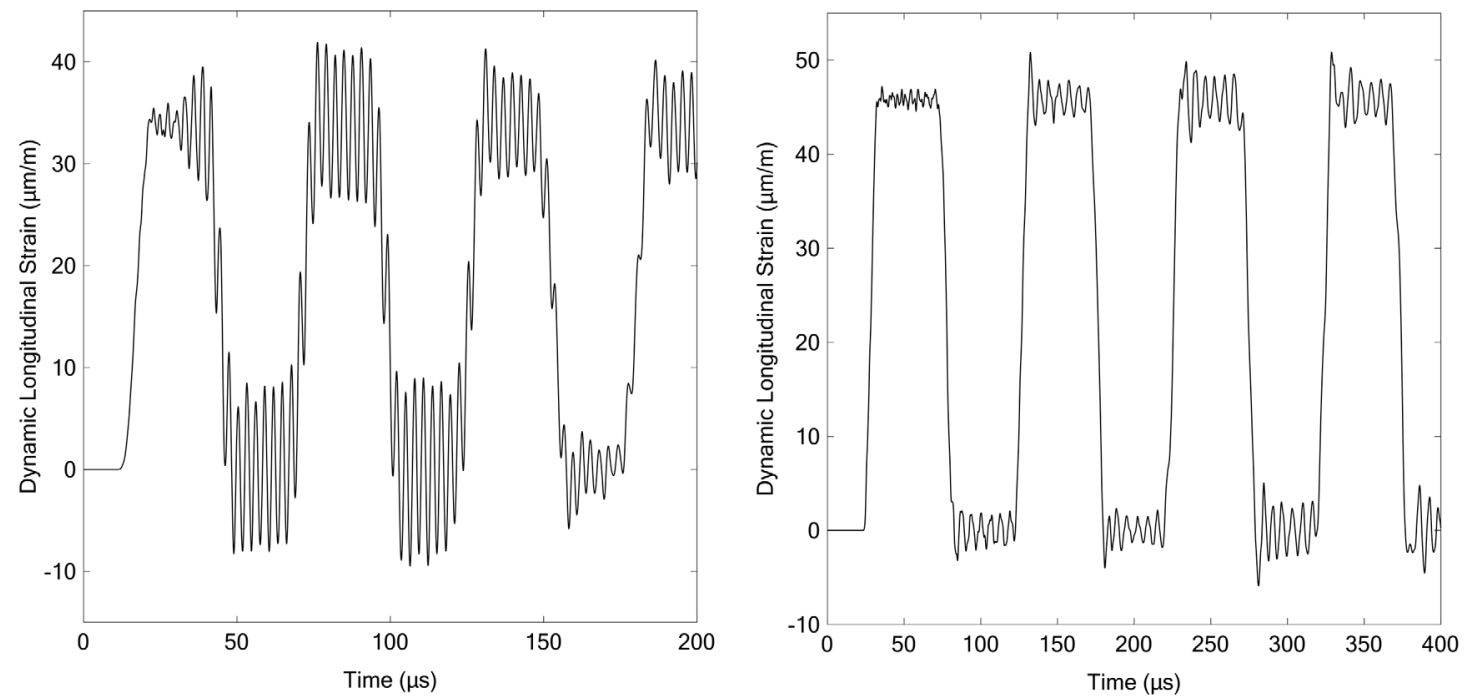

FIG. 7. Dynamic longitudinal strain for rod with a beam sigma of $5 \mathrm{~mm}$ (energy distributed over whole body) for tungsten (left) and graphite (right) at a radius of $0 \mathrm{~mm}$ and a longitudinal position of $\mathrm{L} / 2$.

both material models, however in both cases the amplitude of these components is significantly reduced (from $40 \%$ down to $25 \%$ in the case of tungsten). In this scenario there is no temperature gradient in the radial direction, which could result in the reduction in amplitude observed in the high-frequency components, however radial waves are not completely eliminated due to the relaxation occurring at the rod's surface. The Fourier analysis results also indicate that, in the case of the graphite rod, the second high-frequency harmonic - at approximately $435 \mathrm{kHz}$-is diminished.

The final scenario is that of setting the Poisson's ratio to zero whilst keeping other material and dimensional properties constant, reverting back to a pencil beam with a diameter of $1 \mathrm{~mm}$. The results, shown in Fig. 9 with the corresponding Fourier transform plots in Fig. 10, give an important indication that the high-frequency components observed are indeed due to dispersion of the longitudinal wave and a coupling between radial and longitudinal vibrations, both phenomena being a function of the material's Poisson's ratio. In the case of the tungsten rod, the high-frequency disturbances are essentially eliminated by setting the Poisson's ratio to zero, whilst for the graphite rod some disturbances are still observable but, as can be seen in frequency domain, the high-frequency peaks are no longer tied directly to the radius of the rod, but instead consist of a spectrum of frequencies. The source of these second order effects is possibly a combination of three phenomena: numerical disturbances;
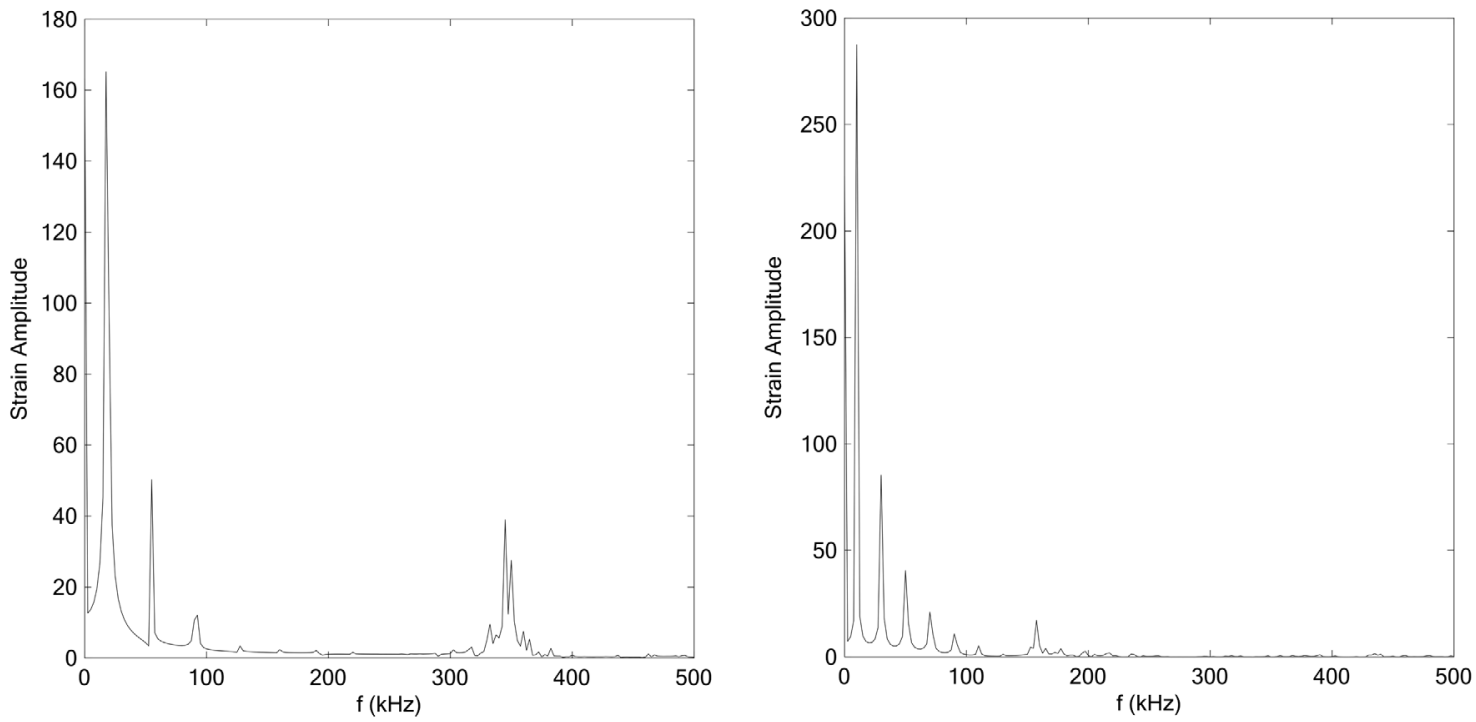

FIG. 8. Fourier analysis of the dynamic longitudinal strain results shown in Fig. 7 for tungsten (left) and graphite (right) rods with a beam sigma of $5 \mathrm{~mm}$ (energy distributed over whole body). 

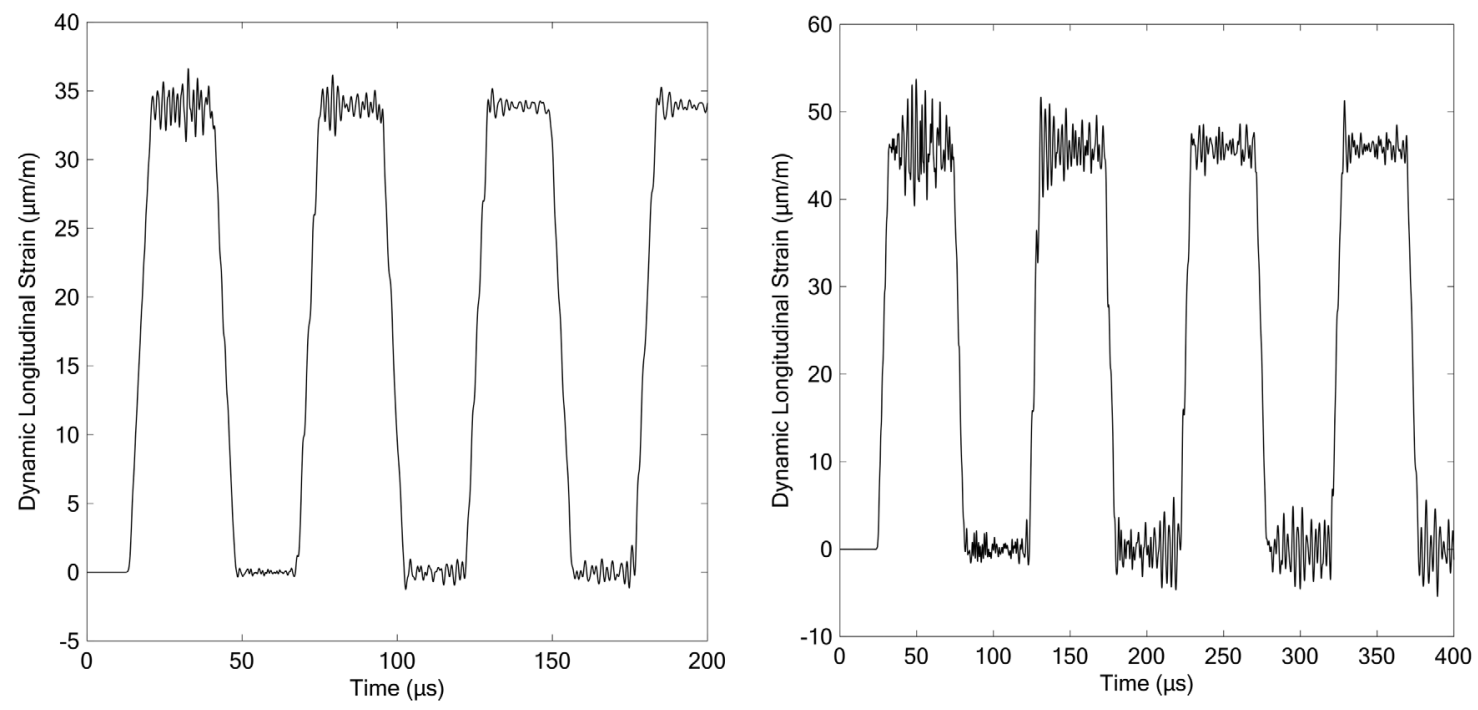

FIG. 9. Dynamic longitudinal strain for rod with $\nu=0$ for tungsten (left) and graphite (right) at a radius of $0 \mathrm{~mm}$ and a longitudinal position of $\mathrm{L} / 2$.

surface waves-such as Rayleigh waves-inducing a longitudinal motion of the surface (and beneath the surface at smaller amplitudes); or modal conversion of purely expansive radial waves to shear waves, thus acting longitudinally. The latter case is similar to the seismological phenomenon where $\mathrm{p}$-waves are partially converted to s-waves upon reflection at an interface [14]. Numerical errors can be considered negligible as in the other scenarios considered, whilst the effects of surface waves can be investigated by taking results at different radial positions. For the scenario considered, the high-frequency components on the dynamic longitudinal strain results at the surface of the rod have a similar amplitude to the results at the core, indicating that the distortion is not a result of surface waves (which decay exponentially with depth from the surface). Further studies are required to better understand such second order effects and shed light on the phenomena in play.

As described by Carra [7], waves propagating radially from the rod's core weakly influence the axial dynamic response when compared to the dispersive effects related to the presence of free lateral surfaces.

In the case of dispersion, high-frequency waves follow the main rectangular wave and thus should appear following its arrival. In the modeled scenarios, taking Figs. 5 and 7 as examples, one can see that the high-frequency
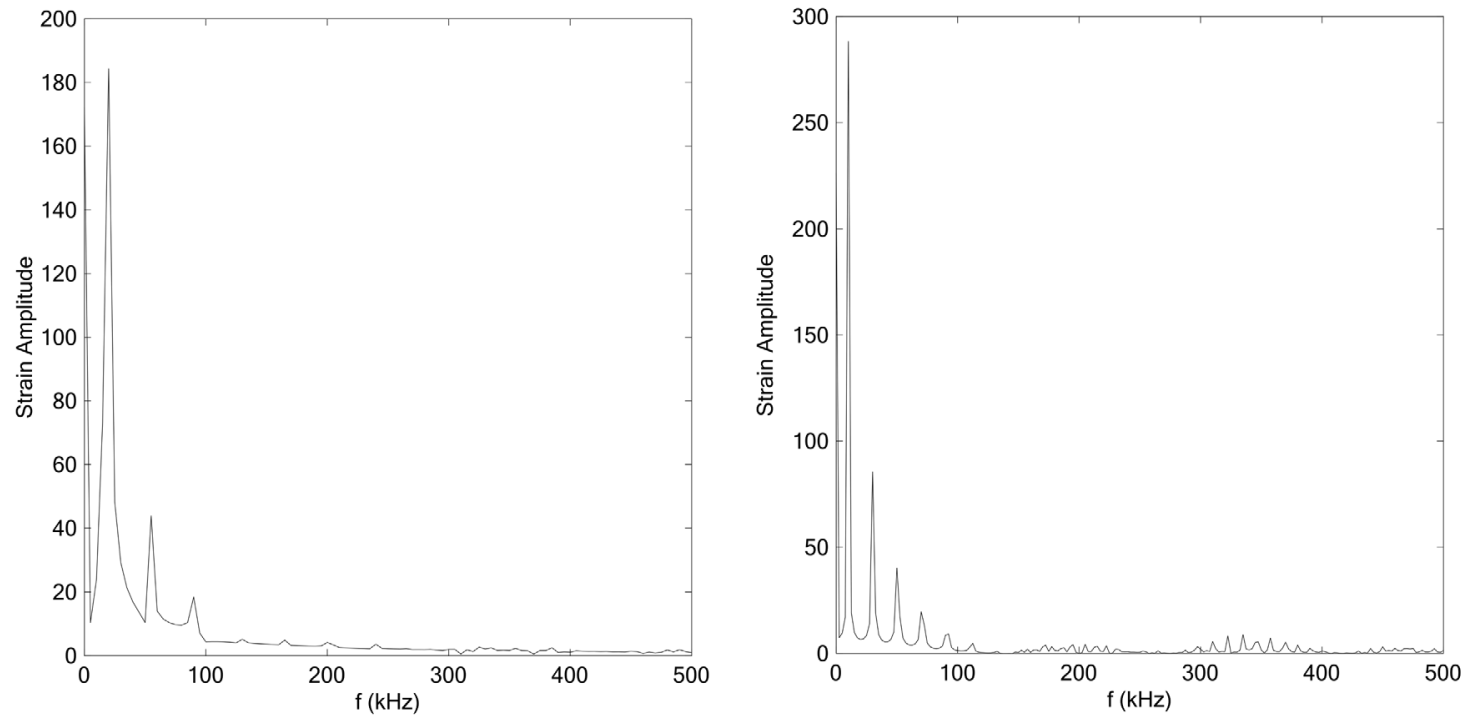

FIG. 10. Fourier analysis of the dynamic longitudinal strain results shown in Fig. 9 for tungsten (left) and graphite (right) rods with Poisson's ratio $\nu$ set to 0 . 
oscillations are observable in the first ramp. At this point, the coupling between radial and longitudinal waves through the Poisson's ratio has already occurred, due to the short time period of the radial waves (approximately $2.85 \mu$ s for the tungsten rod, compared to the $56 \mu$ s period of the longitudinal wave) which only have to travel across the rod's cross section. The results show that these highfrequency oscillations initially have a very small amplitude, which only becomes prominent once the longitudinal wave plateaus at the maximum stress, at approximately $25 \mu \mathrm{s}$ in the case of tungsten.

This behavior is what would be expected if dispersion is the dominating phenomenon, with slower, high-frequency waves following the main longitudinal wave, appearing following its propagation along the cylindrical rod. It can thus be concluded that dispersion is the principal source of high-frequency disturbances, whilst the coupling between radial and longitudinal waves through the Poisson's ratio can be seen to contribute to a much lesser extent in the initial stages of wave propagation. As mentioned, other second order phenomena, including effects related to surface waves, are also possible contributors to the observed disturbances and are not dependent on the material's Poisson's ratio.

\section{NUMERICAL MODELING-COMPARISON BETWEEN THERMOSTRUCTURAL ANALYSIS AND HARMONIC RESPONSE ANALYSIS}

The results presented so far in this study indicate that there are two parameters affecting the dynamic response of a slender rod subject to a rapid internal energy deposition, mainly: (i) the input spectrum imposed on the system due to the initial thermal pulse- this forces the system to oscillate in a range of frequencies depending on the input, which mainly depends on beam parameters and how the beam interacts with the rod; (ii) the harmonic response of the system-this is the range of frequencies at which the system is free to oscillate, depending on the rod dimensions, material properties, and boundary conditions.

This section of the study aims to highlight the effects of the input spectrum on the thermomechanical response, and eventually compare the results with results from modal analyses conducted in ANSYS, discussing the similarities and differences between the two.

The same numerical model presented in the first part of the study is initially used to determine a relationship between the radius of the rod and the frequencies and amplitudes observed, retaining a length of $125 \mathrm{~mm}$, a pulse duration of $7.2 \mu \mathrm{s}$, a beam diameter of $1 \mathrm{~mm}$, and a total energy density of $1000 \mathrm{~J} / \mathrm{cm}^{3}$ at the end of the pulse. The rod radius is varied in a range from 2.5 to $7 \mathrm{~mm}$ in $0.5 \mathrm{~mm}$ steps, maintaining all other parameters constant. This section focuses solely on the material model for graphite, presented in the previous section in Table I. Graphite is widely used in accelerator technology and is one of the materials tested in the HRMT36 experiment, as well as being a constituent in various novel composites developed for use in beam-intercepting devices at CERN, such as molybdenum graphite [15].

The maximum temperature, dependent on the energy deposited and beam sigma, and longitudinal period, dependent on the material properties and length of the rod, are both common for all rod radii. The defined equations give results for a maximum temperature increase of $509^{\circ} \mathrm{C}$ in the heated region, and a longitudinal wave period $t_{L}$ equal to $98.5 \mu$ s (for a longitudinal frequency of $10.2 \mathrm{kHz}$ ). The final uniform temperature, reference stress, and thermal diffusion time are dependent on the total volume, and therefore the radius, of the rod. The analytically computed results for these values are shown in Table III for different radii. The results for the dynamic longitudinal strain following thermomechanical simulations for a range of radii from 2.5 to $4.5 \mathrm{~mm}$ are shown in Fig. 10 .

Ignoring the observed disturbances, analytical and simulation results can be seen to be in good agreement when comparing the results for dynamic longitudinal strain $\left(2 \varepsilon_{\text {ref }}\right)$ in Table III with the peak dynamic strain values displayed in Fig. 11. The maximum strain decreases accordingly with an increase in radius, whilst the longitudinal period is constant at $98.5 \mu \mathrm{s}$ for all rods. The highfrequency component is superimposed on the longitudinal wave as in the previous section, and can be seen to vary in both amplitude and frequency with a change in radius. Figure 12 shows the results of a Fourier analysis on the dynamic longitudinal strain results for five rod dimensions, as well as a zoomed in view on the same plot for all dimensions tested. The Fourier analysis aids in identifying the various frequencies making up each waveform. The underlying longitudinal signal-due to its square-wave shape - is made up of odd harmonics only, which range from the underlying base frequency of $10.2 \mathrm{kHz}$ up to the 11th harmonic (approximately $112 \mathrm{kHz}$ ) for all cases, at

TABLE III. Final uniform temperature increase, reference stress and strain, and thermal diffusion time for rods with radii 2.5-7 $\mathrm{mm}$. Values in bold (range $2.5-4.5 \mathrm{~mm}$ radius) relate to the maximum dynamic longitudinal strain, displayed in Fig. 11.

\begin{tabular}{lccccl}
\hline \hline $\begin{array}{l}\text { Radius } \\
(\mathrm{mm})\end{array}$ & $\begin{array}{c}\mathrm{T}_{f} \\
\left({ }^{\circ} \mathrm{C}\right)\end{array}$ & $\begin{array}{c}\sigma_{\text {ref }} \\
(\mathrm{MPa})\end{array}$ & $\begin{array}{c}\varepsilon_{\text {ref }} \\
(\mu \mathrm{m} / \mathrm{m})\end{array}$ & $\begin{array}{c}2 \varepsilon_{\text {ref }} \\
(\mu \mathrm{m} / \mathrm{m})\end{array}$ & $t_{d}(\mathrm{~s})$ \\
\hline 2.5 & 20.37 & 1.05 & 91.30 & $\mathbf{1 8 2 . 6 1}$ & 0.2 \\
3.0 & 14.14 & 0.73 & 63.65 & $\mathbf{1 2 7 . 3 0}$ & 0.29 \\
3.5 & 10.39 & 0.54 & 46.78 & $\mathbf{9 3 . 5 7}$ & 0.4 \\
4.0 & 7.96 & 0.41 & 35.83 & $\mathbf{7 1 . 6 5}$ & 0.52 \\
4.5 & 6.29 & 0.33 & 28.26 & $\mathbf{5 6 . 5 2}$ & 0.66 \\
5.0 & 5.09 & 0.26 & 22.96 & 45.91 & 0.81 \\
5.5 & 4.21 & 0.22 & 18.96 & 37.91 & 0.99 \\
6.0 & 3.54 & 0.18 & 15.91 & 31.83 & 1.18 \\
6.5 & 3.01 & 0.16 & 13.57 & 27.13 & 1.38 \\
7.0 & 2.6 & 0.13 & 11.65 & 23.30 & 1.6 \\
\hline \hline
\end{tabular}




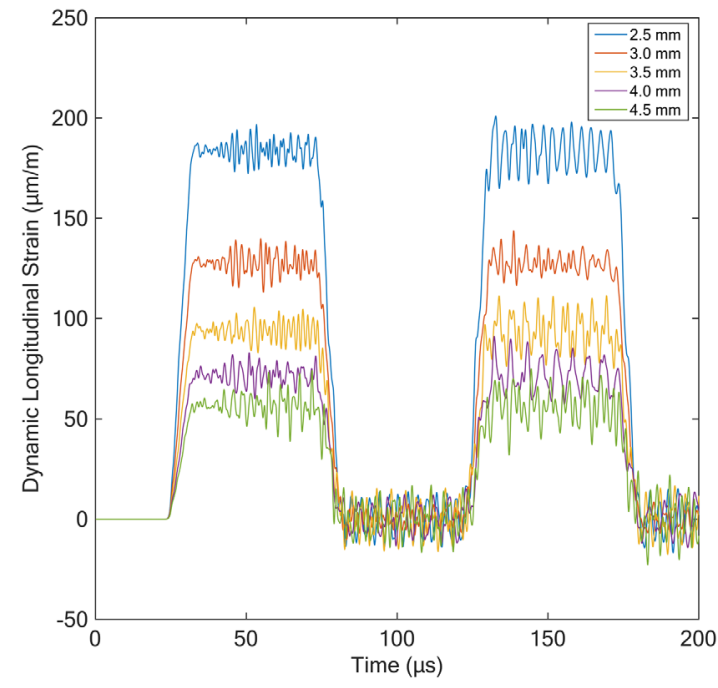

FIG. 11. Dynamic longitudinal strain for graphite rods with radius ranging from 2.5 to $4.5 \mathrm{~mm}$ at a radius of $0 \mathrm{~mm}$ and a longitudinal position of $L / 2$.

which point the amplitude becomes negligible compared to the higher frequency disturbances.

For each case study, i.e. for different radii, there are respective high-frequency radial components, increasing in frequency with a reduction in radius. The radial components are also made up of multiple harmonics, for example for a radius of $6.5 \mathrm{~mm}$ resonant frequencies are observed at 122 and $334 \mathrm{kHz}$. It is also interesting to note that there is a discrepancy between radial harmonic results obtained from the thermostructural analysis and those obtained analytically through Eq. (11). Differences between numerical and analytical solutions are observable at smaller radii (and thus higher frequencies). This follows the considerations made by Graff [6] with regards to the breakdown of the classical wave theory. At low frequencies the wave velocity is close to the ideal velocity and therefore the analytical model correctly predicts the frequency, while with an increase in frequency, as a result of a decrease in radius, the discrepancy between the predicted and actual wave velocity (and in turn that between the predicted and actual frequency) increases due to wave dispersion.

At this point it is also possible to identify a pattern in the frequency spectrum which helps to decouple the effects of the input spectrum and the harmonic response. Displaying all thermostructural analysis results for dynamic longitudinal strain in a graphite rod (for the radius range from 2.5 to $7 \mathrm{~mm}$ ) on the same plot reveals that there are periodic frequencies at which the amplitude is zero or close to zero. For the scenarios tested, this occurs at approximately every $140 \mathrm{kHz}$, separating frequencies in batches. This is a result of the input spectrum from the thermal pulse the system is subject to [16]. The Fourier transform of a rectangular pulse having a pulse duration $\tau=7.2 \mu \mathrm{s}$ and an arbitrary amplitude $A=1$ can be expressed by

$$
G(f)=\frac{\sin (\pi f \tau)}{\pi f}
$$

Figure 13 shows the Fourier transform result for longitudinal strain for all rod radii simulated, plotted along with the absolute values of the scaled input spectrum for a rectangular pulse according to Eq. (13).

The width of each batch of frequencies in Fig. 13 is $139 \mathrm{kHz}$, equal to the inverse of the pulse duration. The input spectrum is characterized by large amplitudes at lower frequencies, which drop quickly to the first

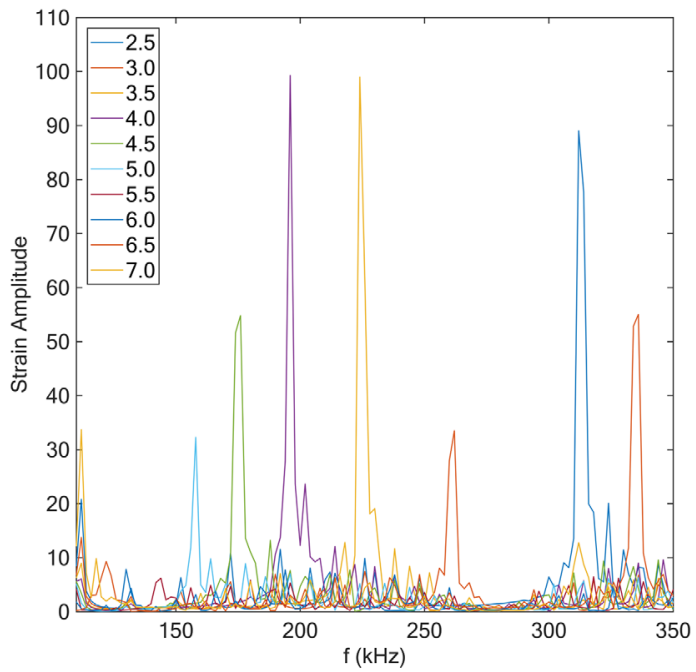

FIG. 12. Fourier analysis for the longitudinal strain at a radius of $0 \mathrm{~mm}$ and a longitudinal position of $L / 2$ for graphite rods with radii in the range 2.5 to $4.5 \mathrm{~mm}$ (left) and zoomed in view in the range $100-350 \mathrm{kHz}$ for all radii modeled (right). For all rod radii, frequency peaks up to approximately $112 \mathrm{kHz}$ correspond to odd longitudinal wave harmonics. Beyond this frequency, longitudinal harmonics are negligible in amplitude when compared to the high-frequency radial harmonics. 


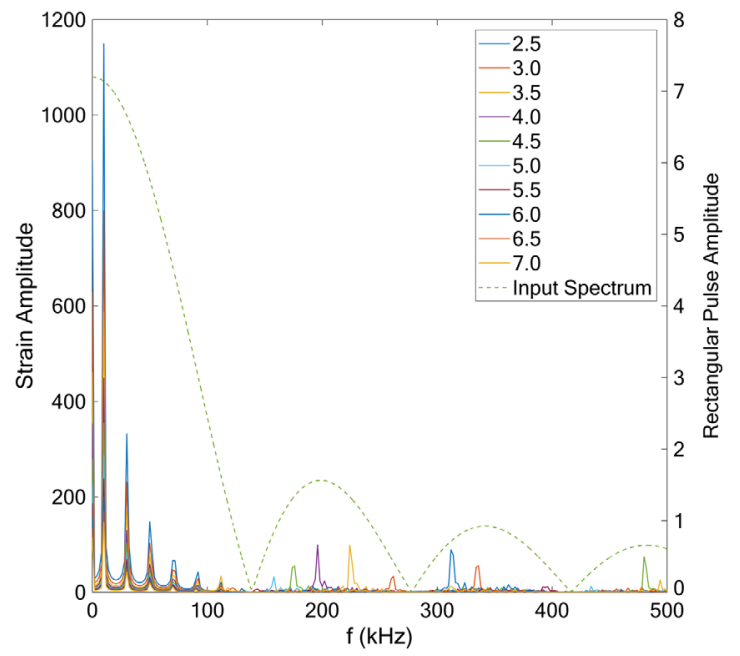

FIG. 13. Fourier analysis for the longitudinal strain for graphite rods with radii in the range 2.5 to $7 \mathrm{~mm}$ with superimposed rectangular pulse spectrum (dotted), showing periodic minima at $139 \mathrm{kHz}$ intervals.

minimum, with higher frequency batches having progressively lower maximum amplitudes.

As mentioned at the beginning of this section, the second component of the dynamic response observed in the thermostructural analyses is related to the harmonic response of the system, corresponding to the longitudinal and radial resonant frequencies. As the material properties and boundary conditions are constant for all modeled scenarios in this section, the harmonic response is solely dependent on the rod dimensions.

Harmonic response analyses are used to predict the sustained dynamic behavior of a structure subject to a load, helping to identify resonant frequencies excited by forced vibrations. The problem can be modeled in ANSYS by performing a modal analysis with a rod subject to a radial force, representing the thermal load due to a particle beam impact pushing from the center of the rod outwards. Due to the material's Poisson's ratio, the rod is still excited longitudinally at low (longitudinal) resonant frequencies and high (radial) frequencies.

Modal analyses are typically less computationally expensive than full transient thermostructural analyses, hence this section sets out to investigate whether the results achieved with a thermostructural analysis can also be attained through the harmonic response spectrum obtained from a modal analysis. The final output of the system - the dynamic longitudinal strain in this case-is a result of the convolution of the input signal and the harmonic response function of the system. One would therefore expect that the result of this multiplication is similar to the results achieved from performing a thermostructural analysis of a beam impact.

The results of multiplying the input spectrum for a rectangular pulse with the axial strain results of a harmonic response simulated in an ANSYS modal analysis, for a rod with a length of $125 \mathrm{~mm}$ and various radius dimensions, are shown in Fig. 14 (left). This can be compared to results from thermostructural simulations, shown in the same figure (right). For both cases, the odd longitudinal wave harmonics dominate at low frequencies and diminish as the first minimum is reached at $139 \mathrm{kHz}$. The modal analysis can be seen to predict well the frequencies in the range of 100 to $500 \mathrm{kHz}$, however the amplitude of the highfrequency harmonics can be seen to be overestimatedfor the $4 \mathrm{~mm}$ radius rod, the harmonic analysis predicts a peak at approximately $200 \mathrm{kHz}$ having an amplitude of $38 \%$ of the base longitudinal harmonic, whilst the

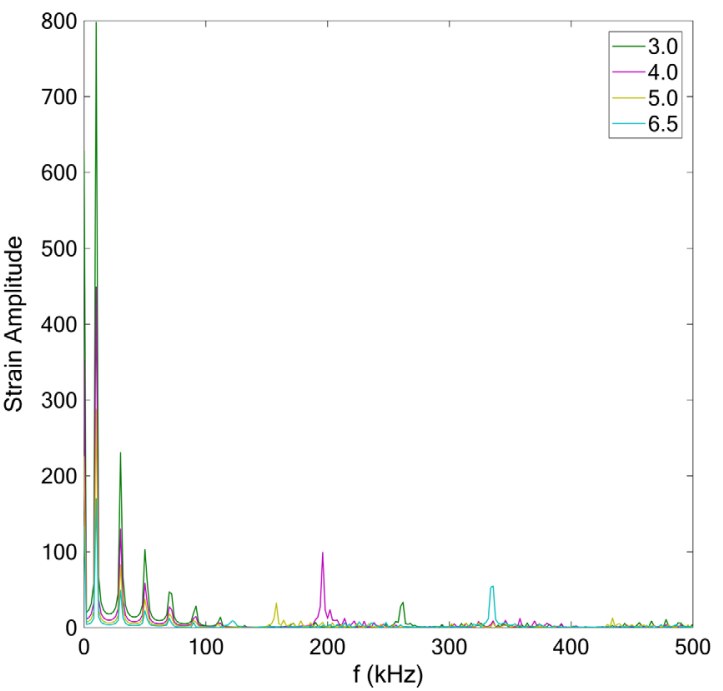

FIG. 14. Expected output of the system (rectangular pulse input multiplied by simulated harmonic response) with damping for graphite rods with length $125 \mathrm{~mm}$ and radii in the range 3 to $6.5 \mathrm{~mm}$ (left), and Fourier transform of thermostructural simulation results for graphite rods with length $125 \mathrm{~mm}$ and radii in the same range (right). 
thermostructural simulation shows a peak at the same frequency having an amplitude of only $22 \%$ of the base harmonic.

The results thus indicate that, while modal analyses can certainly be useful in determining rod geometries which minimize high-frequency components, the analyses need to be fine-tuned further to predict correctly the amplitude of distortional components. This can be done by scaling the amplitudes of the input spectrum and harmonic response to the reference strain, refining the damping parameter, and possibly performing an inverse Fourier transform to obtain the result in the time domain. This could present an alternative to easily identify radial frequencies and, more importantly, their amplitude relative to the longitudinal wave amplitude, in turn making it possible to predict and mitigate these effects by varying the geometry of the rod such that these harmonics are at frequencies which coincide with a minimum in the input pulse spectrum, or else varying the pulse duration to vary the position of these minimums.

Modal analyses could then be used to strike a balance between the speed of analytical solutions-which are used first, when available, to compute quick approximations and parameter optimization - and the customization achievable with full-scale thermomechanical simulations.

\section{CONCLUSIONS}

Numerical models presented in this study are used to investigate the propagation of longitudinal waves in slender rods subject to particle beam impacts, a topic of great interest in accelerator technology, especially with regards to beam-intercepting devices. The study considers a slender cylindrical rod, simply supported and free to expand, subject to a rapid internal temperature increase. The problem is modeled numerically using two methods, the first being a sequentially coupled thermomechanical analysis, and the second based on a modal analysis.

Analytical models considered neglect lateral inertia effects on the longitudinal waveform, however the numerical analyses performed illustrate that these effects, a result of dispersion of the longitudinal wave and to a lesser degree Poisson's ratio effects due to radial wave oscillations, can be included in numerical models and are clearly observable in results. An adequate time step is thus required to ensure that these effects are observable in the analysis results.

Thermomechanical analyses performed show that the dynamic response of a slender rod subject to a rapid internal energy deposition is dependent on the input spectrum of the initial thermal pulse and on the harmonic response of the system. Following this observation, modal analyses are presented and compared to their thermomechanical counterparts, indicating that modal analyses can be used to quickly identify radial harmonics and approximate their amplitude relative to the longitudinal wave amplitude.

The models presented are used to investigate factors affecting the observed radial components, both in terms of frequency and amplitude. This is of particular interest in preparation for experiments such as those conducted at the HiRadMat facility at CERN, where these effects can be manipulated as required by varying parameters such as the rod dimensions and beam parameters.

Other results presented in the study, including observed phenomena such as beating and second order effects in longitudinal waves, could also be the basis for further study in future publications.

\section{ACKNOWLEDGMENTS}

The research leading to these results has received funding from the European Commission under the FP7 Research Infrastructures project EuCARD-2, Grant agreement No. 312453.

[1] A. Bertarelli, Beam-induced damage mechanisms and their calculation, CERN Yellow Reports, 2016, Vol. 2, p. 159, URL: https:/e-publishing.cern.ch/index.php/CYR/article/ view/234.

[2] H. Bargmann, Dynamic response of external targets under thermal shock, CERN Technical Note No. LAB II/BT/Int/ 73-3, 1973.

[3] P. Sievers, Elastic stress waves in matter due to rapid heating by an intense high-energy particle beam, CERN Technical Note No. LAB II/BT/74-2, 1974.

[4] A. Bertarelli, A. Dallocchio, and T. Kurtyka, Dynamic response of rapidly heated cylindrical rods: Longitudinal and flexural behavior, ASME J. Appl. Mech. 75, 031010 (2008).

[5] M. Cauchi, R. W. Assmann, A. Bertarelli, F. Carra, L. Lari, A. Rossi, P. Mollicone, and N. Sammut, Thermomechanical assessment of the effects of a jaw-beam angle during beam impact on Large Hadron Collider collimators, Phys. Rev. ST Accel. Beams 18, 021001 (2015).

[6] K. F. Graff, Wave Motion in Elastic Solids (Dover Publications Inc., New York, 1991), pp. 116-121.

[7] F. Carra, Thermomechanical response of advanced materials under quasi instantaneous heating, Ph.D. thesis, Politecnico di Torino, Italy, 2017, pp. 110-119.

[8] L. Pochhammer, On the propagation velocities of small oscillations in an unlimited isotropic circular cylinder, J. Pure Appl. Math. 81, 324 (1876).

[9] I. Efthymiopoulos, C. Hessler, H. Gaillard, D. Grenier, M. Meddahi, P. Trilhe, A. Pardons, C. Theis, N. Charitonidis, S. Evrard, H. Vincke, and M. Lazzaroni, HiRadMat: A new irradiation facility for material testing at CERN, in Proceedings of the 2nd International Particle Accelerator Conference, San Sebastián, Spain (EPS-AG, Spain, 2011).

[10] SGL Group, Sigrafine R4550 Graphite, URL: http:// www.sglgroup.com/cms/_common/downloads/products/ product-groups/gs/tds/iso/SIGRAFINE_TDS-R4550.02 .pdf.

[11] C. Torregrosa, M. Bergeret, E. Berthome, M. Butcher, M. Calviani, L. Gentini, D. Horvath, and J. Humbert, A. Perillo-Marcone, and G. Vorraro, The HiRadMat 27 
experiment: Exploring high-density materials response at extreme conditions for antiproton production, in Proceedings of the 7th International Particle Accelerator Conference, Busan, Korea, 2016 (JACoW, Geneva, Switzerland, 2016).

[12] F. Carra, A. Bertarelli, E. Berthome, C. Fichera, T. Furness, M. Guinchard, L. Mettler, M. Portelli, S. Redaelli, and O. Sacristan de Frutos, The "MultiMat" experiment at CERN HiRadMat facility: Advanced testing of novel materials and instrumentation for HL-LHC collimators, J. Phys.: Conf. Ser. 874, 012001 (2017)..

[13] M. Cauchi, O. Aberle, R. W. Assmann, A. Bertarelli, F. Carra, K. Cornelis, A. Dallocchio, D. Deboy, L. Lari, S. Redaelli, A. Rossi, B. Salvachua, P. Mollicone, and N. Sammut, High energy beam impact tests on a LHC tertiary collimator at the CERN high-radiation to materials facility, Phys. Rev. ST Accel. Beams 17, 021004 (2014).
[14] R. Stewart, J. Gaiser, R. Brown, and D. Lawton, Converted-wave seismic exploration: Methods, Geophysics 67, 1348 (2002).

[15] A. Bertarelli, F. Carra, N. Mariani, and S. Bizzaro, Development and testing of novel advanced materials with very high thermal shock resistance, EuCARD-2 Scientific Report No. CERN-ACC-2014-0306, 2014, URL: https:// cds.cern.ch/record/1973365/files/CERN-ACC-2014-0306 .pdf.

[16] R. Wang, Introduction to Orthogonal Transforms with Applications in Data Processing and Analysis (Cambridge University Press, New York, 2012), p. 135.

Correction: Figure 3 rendered poorly and has been replaced by a revised image, necessitating reformatting of the article. Reference [8] was deleted and subsequent references have been renumbered. 